\title{
26422 - DOSE-RELATED EFFECT OF SEVOFLURANE ON AIRWAY SIZE AND CONFIGURATION
}

\section{Mauro Arrica MD, Mark Crawford, MBBS; Christopher MacGowan, PhD; Shi- Joon Yoo, The Hospital For Sick Children, Toronto, ONTARIO, Canada}

INTRODUCTION: Previous studies suggest that anesthetic concentrations of inhalational anesthetics inhibit upper airway muscle activity in a dose-independent manner.1 Whether this dose-independent relationship applies to changes in airway caliber is unknown. We sought to evaluate the configurational changes that lead to upper airway narrowing during inhalational anesthesia with sevoflurane and to determine whether these changes are dose-independent within a clinically relevant dose range.

METHODS: With REB approval, 15 ASA I or II children undergoing elective MRI of the brain were studied. Anesthesia was induced and maintained with sevoflurane. Patients breathed spontaneously through a Jackson Rees modification of the Ayres T-piece circuit, and a facemask that was kept in position using a head strap. A respiratory bellows was placed around the chest to obtain a waveform of the respiratory circle. Head position was standardized. MRI images were acquired using a 1.5 Tesla scanner. Axial/oblique single shot fast spin echo pulse sequences were used to acquire images at the level of the soft palate (SP), base of tongue (BT), and tip of epiglottis (TE). Image acquisitions were triggered manually by a single investigator in early expiration. Images were acquired at each of three end-tidal concentrations of sevoflurane, 0.5, 1.0, and 1.5 MAC (ageadjusted), administered in random sequence. Randomization was achieved using a random number table. At least 15 min was allowed for equilibration of inspired and alveolar partial pressures after each change in sevoflurane concentration. Airway images were analyzed by a blinded investigator who was unaware of the MAC concentration. Airway cross-sectional area (CSA), anteroposterior, and transverse dimensions were determined using image-analysis software, taking the average of three successive measurements. Repeated-measures ANOVA and the Student-Newman-Keuls post hoc test were used to compare airway areas and dimensions. $\mathrm{P}<0.05$ was considered statistically significant.

RESULTS: Mean age and weight were $5.3 \pm 2.6 \mathrm{yr}$ and $19.9 \pm 5.9 \mathrm{~kg}$, respectively. Increasing the concentration of sevoflurane was associated with a dose-dependent reduction in airway CSA at each anatomical level (Fig.) $(\mathrm{P}<0.05)$. Increasing the sevoflurane concentration from 0.5 to $1.0 \mathrm{MAC}$ decreased airway CSA by $11 \%$ to $19 \%$, and a further increase to $1.5 \mathrm{MAC}$ resulted in an overall $28 \%$ to $34 \%$ reduction in CSA. For each increase in anesthetic concentration, the percent reduction in CSA at the BT was only slightly greater than that at the SP and TE. At each anatomical level, the reduction in CSA was predominantly due to a decrease in anteroposterior dimension.

DISCUSSION: Increasing the depth of sevoflurane anesthesia resulted in a relatively uniform reduction in caliber across all anatomic levels of the pharyngeal airway. The effect of sevoflurane on upper airway caliber is dose-dependent. 
REFERENCES: 1. Anesthesiology 2002 97: 786-793

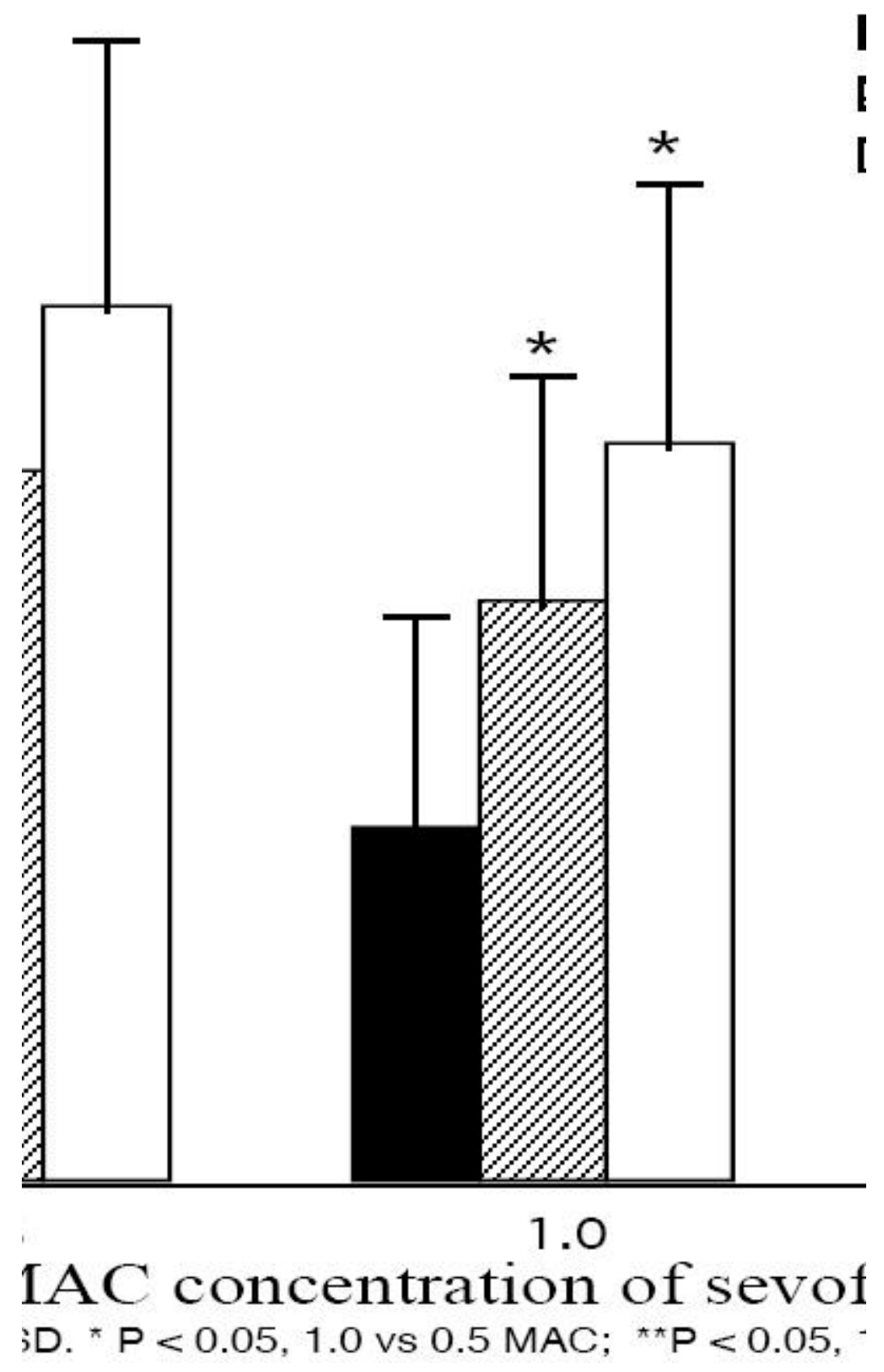

